\title{
Analysis of behavior of slope models for collapsing from stability by shaking table tests under $1 \mathrm{G}$ field
}

\author{
Susumu Nakamura ${ }^{1, *}$ \\ ${ }^{1}$ Nihon University, College of Eng., Civil Engineering Dept., 963-8642 Tokusada, Tamura, Koriyama, Fukushima, Japan
}

\begin{abstract}
The two major subjects were considered based on a result of the shaking experiment of a slope model under $1 \mathrm{G}$ field in this paper. One is the behavior to being unstable from stability of a slope. Second is applicability of Newmark method for the behavior beyond the stable limit behavior. The existing experimental data obtained by shaking table tests of slope models under $1 \mathrm{G}$ were used. The slope models consisted of 3 layers such as surface layer, weak layer and base layer. Among the models, the heights were $1.0 \mathrm{~m}$ for small size model, $2.0 \mathrm{~m}$ for medium size model. The occurrence location of slip surface is restricted in the weak layer. It is found that slip surface is formed by connecting shear cracks which have formed discretely after generating a tensile crack in the top, and that the yield acceleration is the same with the acceleration which acts on a slope when collapsing against a sinusoidal input wave. Furthermore, appropriate consideration is necessary to use the Newmark method for a design because the variation in the estimated value according to the strength characteristic is very large.
\end{abstract}

\section{Introduction}

The evaluation of the response about displacement and the deformation in the unstable state beyond the stable limit is necessary to establish the limit value of either the displacement or the deformation as the evaluation of the stable limit about the slope and the earth structure during earthquake. As the method, the Newmark method is that the theory is not only concise but also a relatively proper result and is used as design standards such as railroad structure [1, 2], road structure [3] and fill dam [4]. The Newmark method is a method that the movement of the soil block on the slip surface which is estimated by stable analysis based on the equilibrium of forces is calculated easily as rigid plastic behavior. As for the Newmark method, a correction which considered materials properties and response property of the ground is carried out by many researchers since Newmark [5] proposed, and validation is performed by experiments. However, by the verification of Newmark method based on the experiment of the conventional slope model by using the shaking table tests under $1 \mathrm{G}$ field, the evaluation about the precision and the applicability have not carried out enough.

On the other hand, it is pointed out by Shinoda et al [6] that the collapse modes are classified into the following three modes based on the result obtained by shaking table tests of three slope models. The slope model consists of three layers : surface layer, foundation layer, weak layer in which generates slip surface. The first form is Quick sliding which will slip off immediately after slip surface was generated. The second form is Slow sliding where deformation progresses during shaking and becomes stable behind the shaking. The last form is Quick sliding after sliding where Slow sliding slips off during progress. Furthermore, among three collapse forms, attention is paid to two experiments that not only Slow sliding but also Quick sliding after sliding were generated, and movement value of surface layer that is estimated by the Newmark method is compared with the experimental value, and the usefulness was shown based on the comparison.

A idea of the stable limit by which it is assumed that a slope was a stable state when movement value of the surface layer was smaller than the limit value based on the result, was proposed. The validity of the displacement estimate by the Newmark method is performed against only the experimental results of two slope models. From the viewpoint for verifying precision as well as applicability, it is not sufficient. For the three experiments, the state that collapse starts, i.e. the state that slip surface is generated, is evaluated based on the vertical displacement in surface layer obtained after generating tension crack in weak layer at the shoulder of the slope model.

In the paper, at first, the analysis in the process to the unstable state of surface layer from the formation of the slip surface which depend on the collapse mode is carried out based on the experiments including not only the slope models which is used by Shinoda et al but also the other slope models $[7,8]$. Then, as the method to evaluate movement value of the surface layer on the slip surface, the applicability of Newmark method is examined. The

*Corresponding author: s-nak@civil.ce.nihon-u.ac.jp 


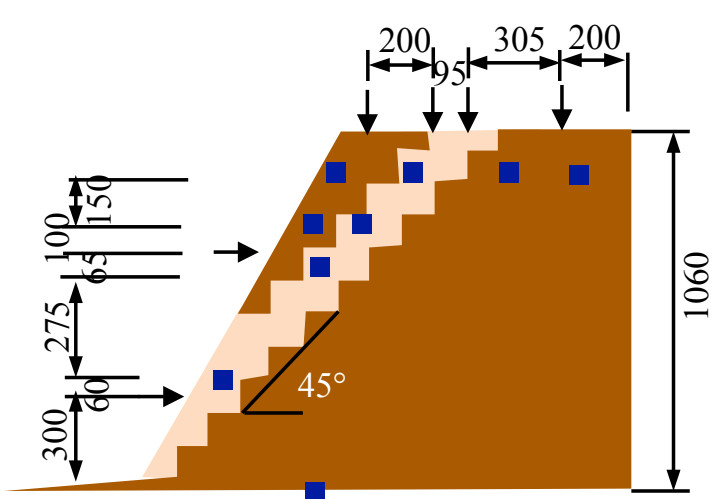

a) case 5

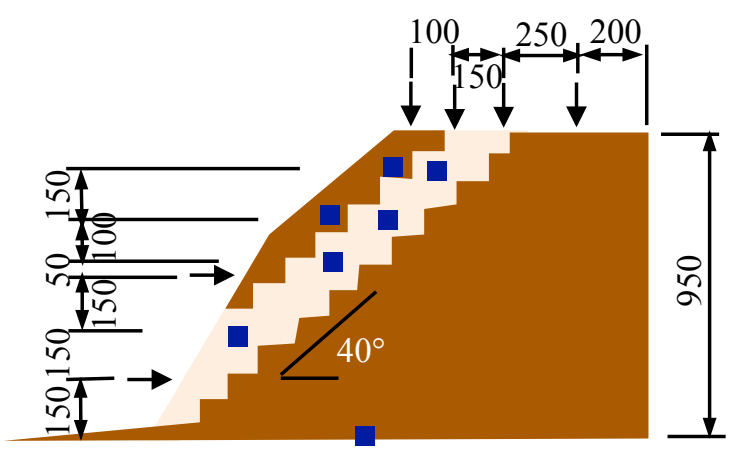

c) case 7,14

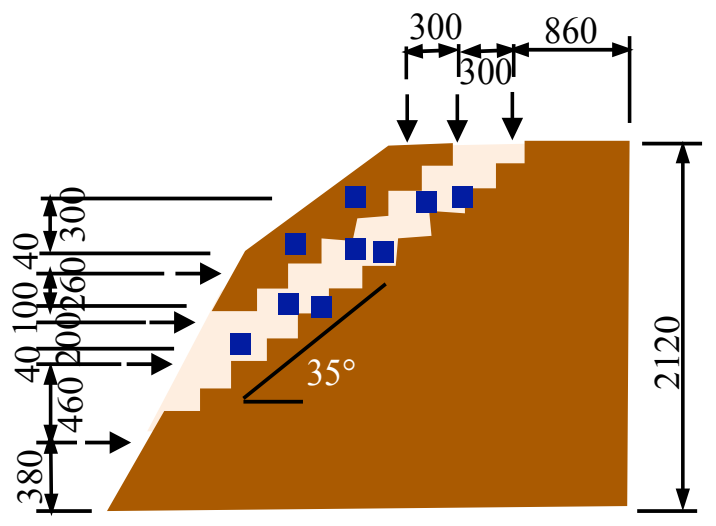

e) case 10,11

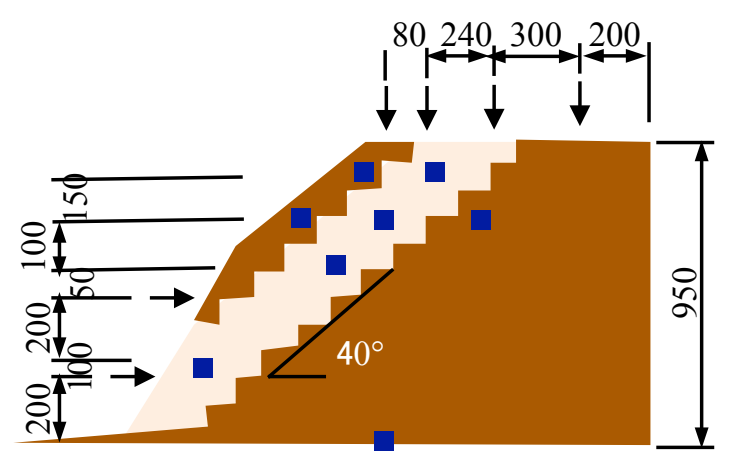

b) case 6

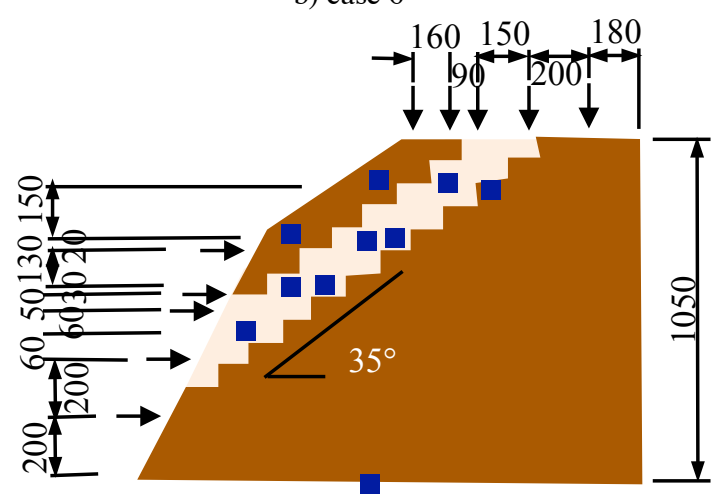

d) case 8,9

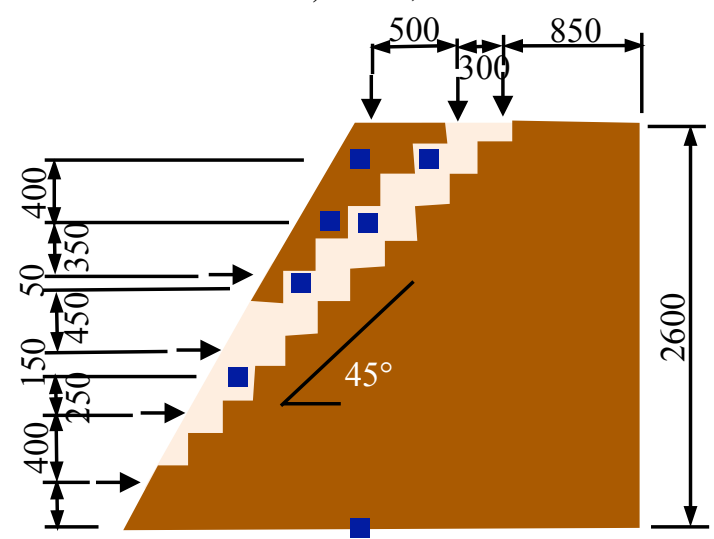

f) case 12,13

Figure 1 Shape of the slope model and configuration of measurement device

(Blue $\mathbf{~ i s ~ a c c e l e r o m e t e r ~ o f ~ h o r i z o n t a l ~ a n d ~ v e r t i c a l ~ c o m p o n e n t , ~ a r r o w ~ i s ~ d i s p l a c e m e n t ~ s e n s o r ) ~}$

existing shaking table tests which are used in this study are performed under $1 \mathrm{G}$ field, and the slope model which consists of three layers : surface layer, base layer, weak layer in which slip surface is generated. High slope model is two kinds, and they are a small slope model of around $1 \mathrm{~m}$, medium-sized slope models of around $2 \mathrm{~m}$.

\section{Outline of existing experiments}

\subsection{Slope model}

The target slope models of the existing experiments are six small slope models and four medium-sized slope models and is in total ten models. Those slope shapes are shown in figure 1. As the shape of the small slope models, the heights are from $0.95 \mathrm{~m}$ to $1.1 \mathrm{~m}$, and the width and the depth are $1.5 \mathrm{~m}, 0.6 \mathrm{~m}$ respectively. As the shape of the medium-sized slope models, the heights are either $2.12 \mathrm{~m}$ or $2.6 \mathrm{~m}$, the width and the depth are $3.0 \mathrm{~m}, 1.0 \mathrm{~m}$ respectively. As shown in figure.1, most of the slope models are comprised of three layers. The surface layer is a sliding block on the slip surface. The slip surface is generated in the weak layer. And there is the base layer below the weak layer. The slope model of case 11 is comprised of two layers of the surface layer and the base layer, and the surface layer is the same material with that of the weak layer of other models. The layer border with the weak layer is not a plane with a stepped interface so that sliding does not occur. In addition, the thickness of the weak layer in the small slope model assumed $80 \mathrm{~mm}$ a standard and did the weak layer thickness of case 6 with $160 \mathrm{~mm}$ that was standard double. As for the weak range 
Table 1 Experimental condition of each case

\begin{tabular}{|c|c|c|c|c|c|c|}
\hline \multirow{2}{*}{$\begin{array}{l}\text { Case } \\
\text { No. }\end{array}$} & \multicolumn{3}{|c|}{ Properties of weak layer } & \multirow{2}{*}{$\begin{array}{l}\text { Presence of } \\
\text { surface layer }\end{array}$} & \multirow[b]{2}{*}{ Size } & \multirow{2}{*}{$\begin{array}{l}\text { Input } \\
\text { wave }\end{array}$} \\
\hline & $\begin{array}{c}\text { Inclination of } \\
\text { weak layer (degree) }\end{array}$ & $\begin{array}{c}\text { Thickness of } \\
\text { weak layer }(\mathrm{mm})\end{array}$ & $\begin{array}{l}\text { Strength } \\
\text { property }\end{array}$ & & & \\
\hline 5 & 45 & 80 & \multirow{8}{*}{$\begin{array}{c}\text { Material } \\
\text { A }\end{array}$} & \multirow{6}{*}{ yes } & \multirow{5}{*}{ small } & \\
\hline 6 & \multirow{2}{*}{40} & 160 & & & & sine \\
\hline 7 & & \multirow{4}{*}{80} & & & & \\
\hline 8 & \multirow{4}{*}{35} & & & & & \\
\hline 9 & & & & & & irregular \\
\hline 10 & & & & & \multirow{4}{*}{ medium } & sine \\
\hline 11 & & - & & - & & \\
\hline 12 & \multirow{2}{*}{45} & \multirow{3}{*}{80} & & \multirow{3}{*}{ yes } & & irregular \\
\hline 13 & & & Material & & & \\
\hline 14 & 40 & & B & & small & sine \\
\hline
\end{tabular}

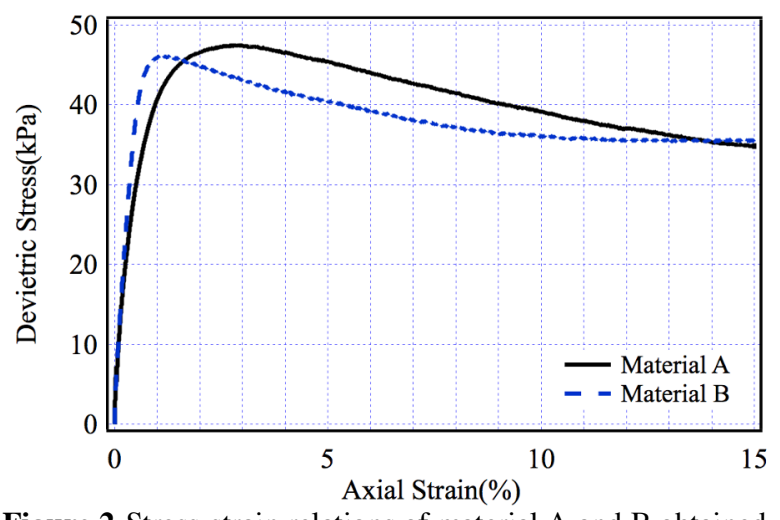

Figure 2 Stress-strain relations of material A and B obtained by triaxial compression tests $\left(\sigma_{c}=10 \mathrm{kPa}\right)$

of medium-sized slope models thickness, $160 \mathrm{~mm}$ is a standard.

As the number of case about slope model the same number with the existing experiments that is referred in this paper is used. Because the evaluation of the process when slip surface is formed in a weak layer and the later deformation behavior are the objective of this paper, the characteristics of the weak layer that had an influence on them are used with the parameter of 10 slope models. The parameter is angle, strength properties, thickness, presence in the surface layer above the weak layer. Furthermore, the model size and input wave are used as the parameter. An experimental case and experimental conditions are shown in Table.1. The angles of the weak layer are three cases of 35 degrees, 40 degrees, 45 degrees. In order to understand the influence that materials difference gave to collapse behavior, two kinds of the materials properties are used. One is the basic characteristic materials A and the other is the materials B that the strain of the peak strength and the strength behavior of the post-peak are different .

The relations between deviator stress and axial strain to the confining pressure $10 \mathrm{kPa}$ obtained from a triaxial compression test of material A, B are shown in figure 2 as a comparative example. Material A was made by adding bentonite and water to silica No.6, and the respective weight ratio was 100:1:10. Material B was made by adding steel grid, slaked lime and water to silica No.8, and

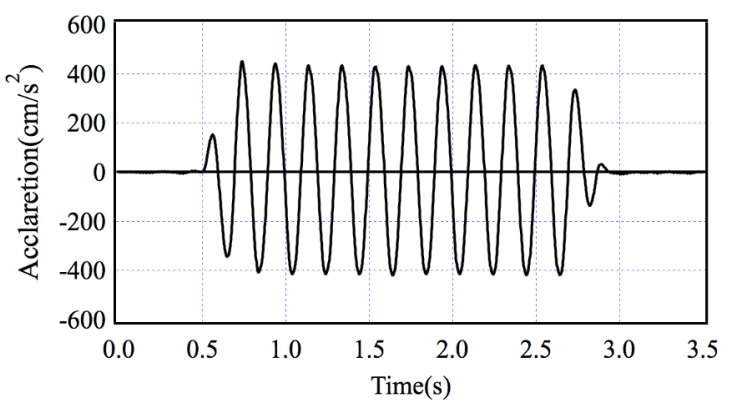

a) Sinusoidal wave

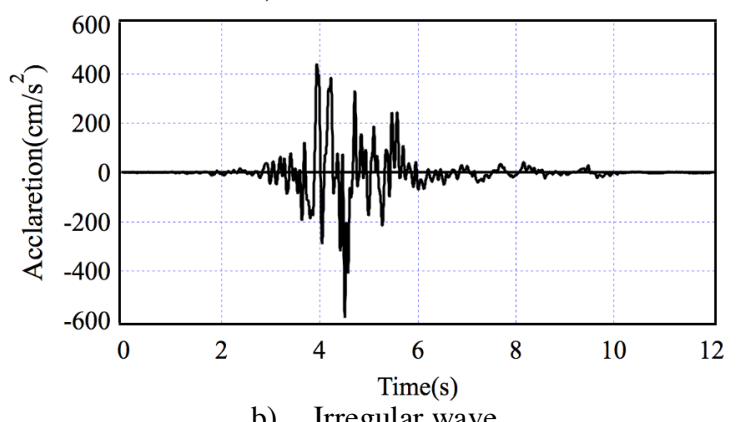

Figure 3 Input wave

the respective weight ratio was 30:70:1:2. For increasing the inertia force of the surface layer, iron sand that unit volume weight is large is used to make the surface layer. In addition, a geo-grid was laid in the surface layer inside to prevent the outer layer from collapsing before shaking. The base layer was made from adding cement to crushed stone by tamping.

\subsection{Input wave and measurement}

The input waves of 2 kinds, the sine wave (10 waves of frequency $5 \mathrm{~Hz}$ ) and the irregular wave were used for shaking. The half wave that adjusted the amplitude with cosine taper is added back and forth of the sine wave so that the amplitude did not increase rapidly. The seismic record observed in Kariwa Kashiwazaki Nuclear Power Plant at Niigata Chuetsu earthquake in 2004 is used for the irregularity wave. An example of input waves on shaking table are shown in figure 3 . As the shaking step, 
Table 2. Shaking step of each case

\begin{tabular}{|c|c|c|}
\hline $\begin{array}{l}\text { Case } \\
\text { No. }\end{array}$ & Input wave & $\begin{array}{c}\text { Amplitude for each step unlit final } \\
\text { shaking step }(\mathrm{Gal})\end{array}$ \\
\hline 5 & \multirow{4}{*}{ Sine } & $100 \rightarrow 200 \rightarrow 300 \rightarrow 400$ \\
\hline 6 & & $100 \rightarrow 200 \rightarrow 300 \rightarrow 400 \rightarrow 500$ \\
\hline 7 & & $100 \rightarrow 200 \rightarrow 300 \rightarrow 400 \rightarrow 500$ \\
\hline 8 & & $100 \rightarrow 200 \rightarrow 300 \rightarrow 400 \rightarrow 500 \rightarrow 600$ \\
\hline 9 & Irregular & $\begin{array}{l}100 \rightarrow 200 \rightarrow 300 \rightarrow 400 \rightarrow 500 \rightarrow 600 \rightarrow \\
700 \rightarrow 800 \rightarrow 900 \rightarrow 900\end{array}$ \\
\hline 10 & \multirow[b]{2}{*}{ Sine } & $\begin{array}{l}100 \rightarrow 150 \rightarrow 200 \rightarrow 250 \rightarrow 300 \rightarrow 350 \rightarrow \\
100 \rightarrow 200 \rightarrow 300\end{array}$ \\
\hline 11 & & $\begin{array}{l}100 \rightarrow 150 \rightarrow 200 \rightarrow 250 \rightarrow 300 \rightarrow 350 \rightarrow \\
100 \rightarrow 200 \rightarrow 300 \rightarrow 400 \rightarrow 450 \rightarrow 500 \rightarrow \\
200 \rightarrow 300 \rightarrow 450\end{array}$ \\
\hline 12 & \multirow{2}{*}{ Irregular } & $50 \rightarrow 100 \rightarrow 200 \rightarrow 200$ \\
\hline 13 & & $50 \rightarrow 100 \rightarrow 200 \rightarrow 200 \rightarrow 300$ \\
\hline 14 & Sine & $100 \rightarrow 200 \rightarrow 300 \rightarrow 400 \rightarrow 500$ \\
\hline
\end{tabular}

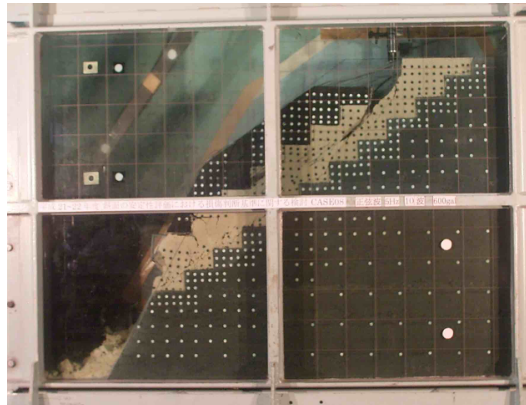

a) case 8 (slow sliding)

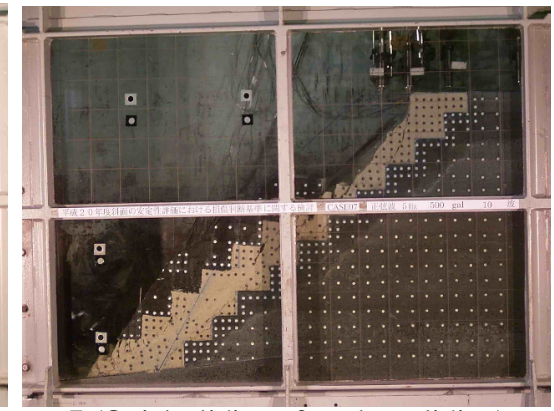

b) case 7 (Quick sliding after slow sliding)
Table 3. Classification of all cases based on collapse mode

\begin{tabular}{|c|c|c|c|}
\hline $\begin{array}{l}\text { Inclination } \\
\text { of weak layer }\end{array}$ & Slow sliding & $\begin{array}{c}\text { Quick } \\
\text { sliding after } \\
\text { slow sliding }\end{array}$ & Quick sliding \\
\hline 35 & $8,9,(\mathbf{1 1})$ & 10 & - \\
\hline 40 & 6 & 7 & $\underline{14}$ \\
\hline 45 & - & - & $5,12, \underline{13}$ \\
\hline & & & \\
\hline & & & \\
\hline & & & \\
\hline & & & \\
\hline & & & \\
\hline & & & \\
\hline & & & \\
\hline
\end{tabular}

Figure 4 An example of three cases of different collapse mode the smallest amplitude of the input wave is 100Gal. The process that the shaking is performed after the amplitude of the input wave is increased $100 \mathrm{Gal}$ is repeated. The shaking process to final shaking of each case is shown in Table 2. The smallest amplitude of case 12, 13 changed to $50 \mathrm{Gal}$. The amplitude of the input wave for case 10,11 is increased 100Gal. After a clear crack appeared in the weak layer, the amplitude of the input wave is decreased $¥$ and the shaking continued until increase of the displacement or collapse occurred.

For measuring the response of the slope, horizontal and vertical displacement sensors as well as accelerometer were installed on the face of slope and the top in the small and medium slope model as shown in figure 1. Furthermore, the estimate of the displacement to occur in the ground using image analytical method used by a similar experiment by Watanabe et al. [9] is carried out. As the method, the target points are set up by every 5 $\mathrm{cm}$ of height and width in a model side of the glass face. Next, the movement of the target points are estimated by the image analyses after taking a picture of the target points by high-speed camera.

\subsection{Collapse mode}

Because the experiments include the experiments that were utilized by Shinoda et al., the three modes, proposed by Shinoda et al., depending on the post behavior after the slip surface was generated in the weak layer are used to arrange the collapse mode. At first, the collapse mode that and the sliding lump including the surface layer and the weak layer on the slip surface slip down on just after the slip surface generates is named as quick sliding. Then, the displacement of the sliding lump gradually increases by shaking, and the collapse mode that the lump becomes a re-stability state after shaking completed is named as slow sliding. Finally, the displacement of the sliding lump on the slip surface gradually increases by shaking, but the collapse mode that slipping down occurs after the displacement of the sliding lump increases rapidly halfway is named as quick sliding after slow sliding. About the behavior of the sliding lump after the slip surface formation about 10 cases, the collapse mode of each case is arranged by considering the relation with the inclination of the weak layer and is shown in Table 3. About case 11 of two layers as the slope models, the collapse mode is arranged as the relations with the inclination of the base layer. In the table, the bold number is the case of the medium slope model, and the number of the blue character is the case of shaking by the irregular wave, the number of the underline is the case using materials B to the weak layer. In addition, the collapse mode of case 11 is expressed as the parenthesis because a toe of slope for slightly collapsed as after generating slow sliding that slip surface is formed near the interface with the base layer. An example about the situation after the last shaking of each collapse mode is shown in figure 4 . In the picture, case 8 is slow sliding, and case 5 is quick sliding. Case 7 is quick sliding after slow sliding. The sliding limp on the slip surface stops after the end of shaking and is stabilized for the case of slow sliding shown in Picture 1 but the sliding lump on the slip surface 


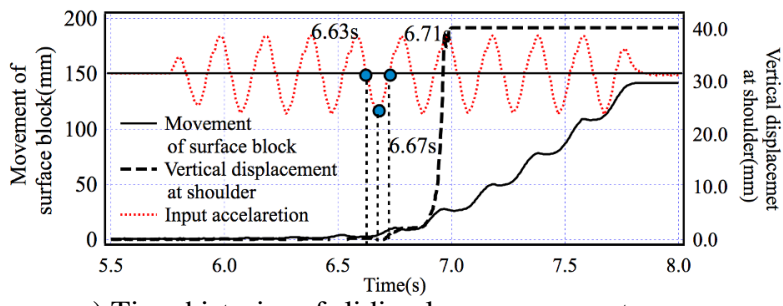

a) Time histories of sliding lump movement

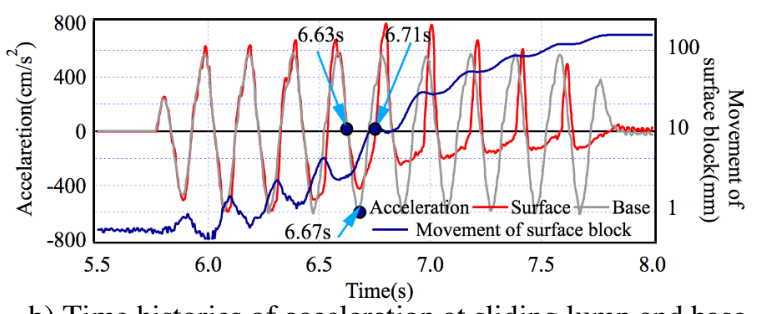

b) Time histories of acceleration at sliding lump and base

Figure 5 Case 8 : sliding lump movement and acceleration at sliding lump and base

( Inclination of weak layer 35 degree, sinusoidal wave )

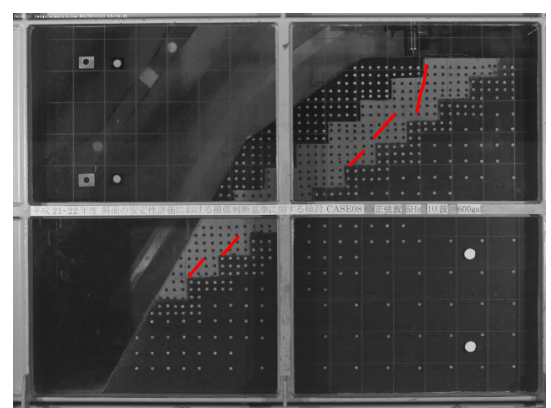

a) $6.63 \mathrm{~s}$

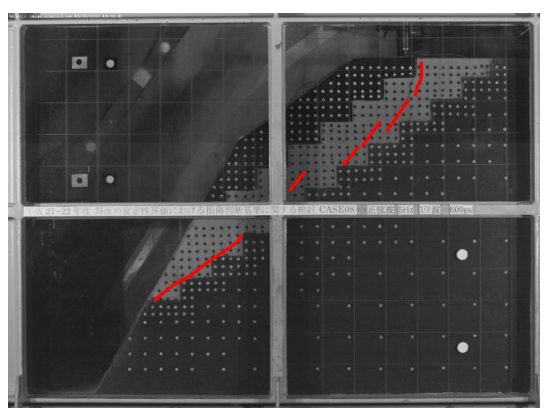

b) $6.67 \mathrm{~s}$

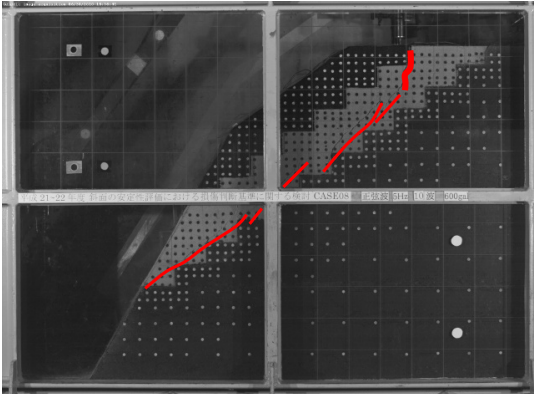

c) $6.71 \mathrm{~s}$

Figure 6 Crack development process of the weak layer for case 8

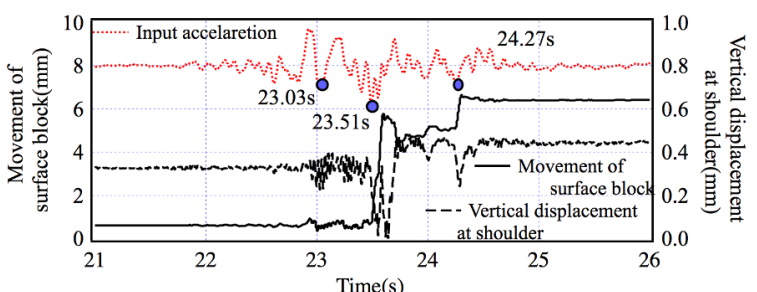

a) Time histories of sliding lump movement

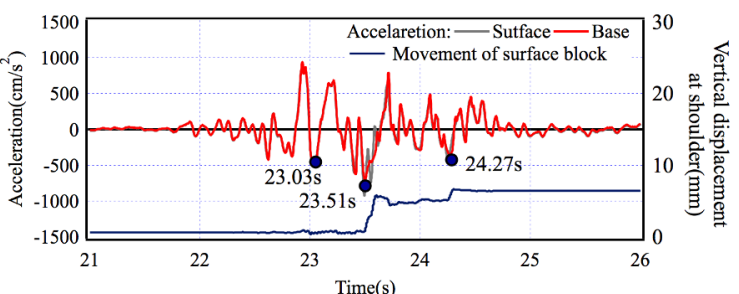

b) Time histories of acceleration at sliding lump and base

Figure 7 Case 9 : sliding lump movement and acceleration at sliding lump and base

( Inclination of weak layer 35 degree, Irregular wave )

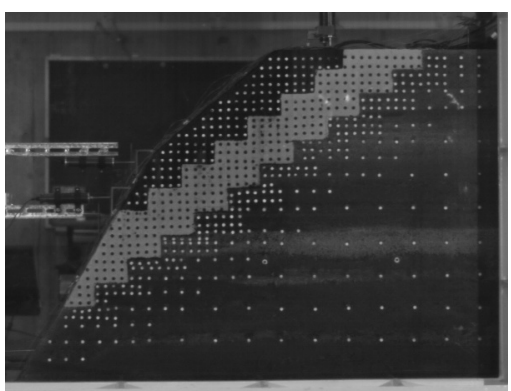

a) $23.03 \mathrm{~s}$

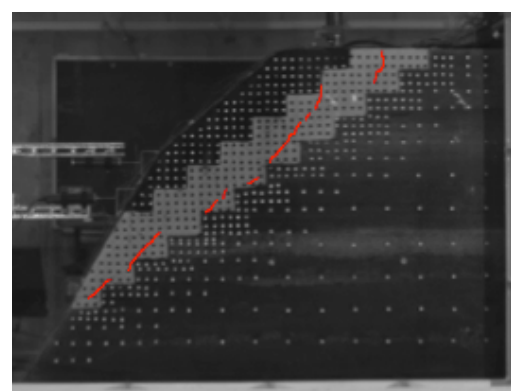

b) $23.51 \mathrm{~s}$

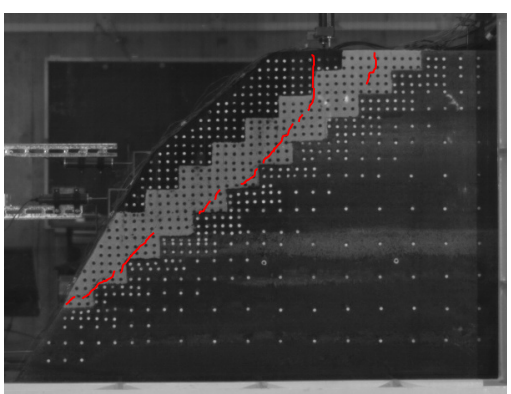

c) $24.27 \mathrm{~s}$

Figure 8 Crack development process of the weak layer for case 9

slips off for the case of quick sliding after slow sliding and quick sliding, and reaches the toe of slope model.

\section{Behavior of formative process of slip surface and sliding lump}

Here, focusing on the quick sliding and the slow sliding as the collapse behavior of the slope model, the representative examples about the relations with a formation process of the slip surface and the behavior of the sliding lump are described.

\subsection{Slow sliding}

As for the results of the last shaking step about 2 cases that the inclination of the weak layer is 35 degrees, case 8 (sinusoidal wave) and case 9 (irregular wave), time histories of the mean value of the movements obtained by the image analyses about 3 target points in surface layer are shown in figures 5a), 7a) and 9a). Time histories of input acceleration at the bottom of the slope model are shown in the same figures. Next, the comparison of the horizontal acceleration time history in the surface layer 


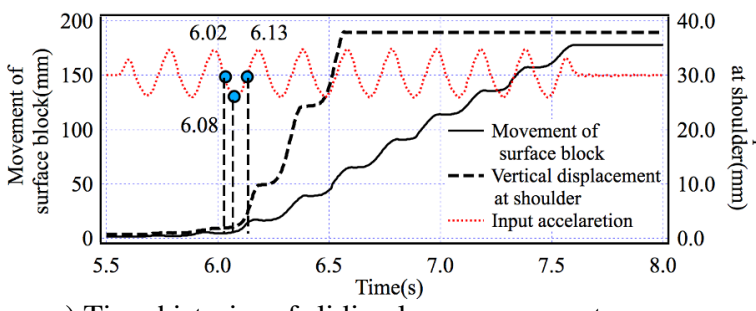

a) Time histories of sliding lump movement

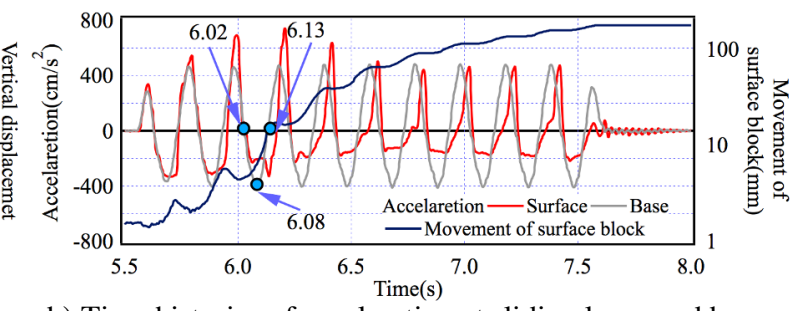
b) Time histories of acceleration at sliding lump and base
and acceleration at sliding lump and base ( Inclination of weak layer 40 degree, Sinusoidal wave )

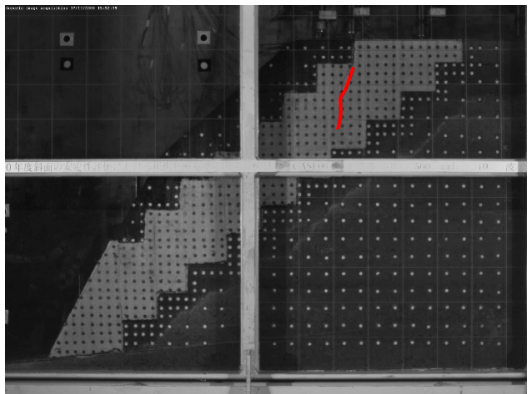

a) $6.02 \mathrm{~s}$

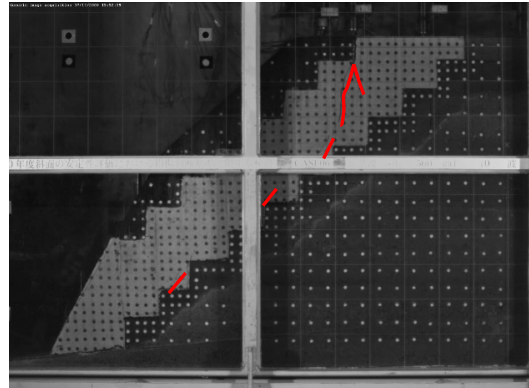

b) $6.08 \mathrm{~s}$

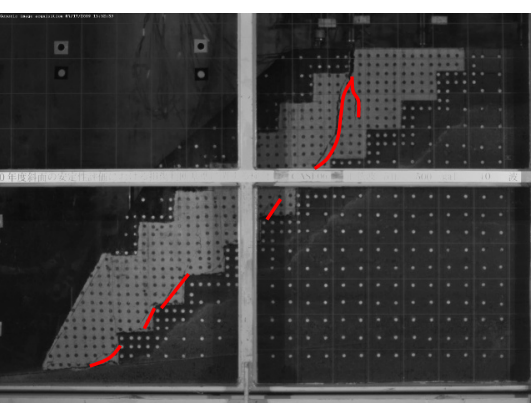

c) $6.13 \mathrm{~s}$

Figure 10 Crack development process of the weak layer for case 6

and that in the base layer is shown in figure 5b), 7b) and $9 b)$. It's shown on each figure together with time history of the movement amount of displacement of the gage point. Time histories of movement shown in previous figures are shown in the same figures. Finally, the crack development process in the weak layer according to the developmental process of the movement of the sliding lump is shown in figures $6,8,10$. The results to the first shaking of $900 \mathrm{Gal}$ is expressed in the previous figures and the photos about case 9, and slip surface seems to be formed after tension crack and shear crack develop. As for a sign of the movement of the target points in the figure, the direction to the toe of the slop model is + . The sign of the acceleration is + for left sides from the right in the slope model shown in figure 5.

At first, about case 8 , focusing on minus acceleration, i.e. the state that the inertial force acts on to the toe direction of the slope. After the movement of the surface layer occurs in the fifth wave (including one tapering portion) as shown in figure 7a), and the movement increased after the sixth wave with the shaking. At 6.63 seconds to go to the fifth wave, a shear crack occurred as well as the tension crack at the shoulder of the slope discretely in the weak layer as shown in figure 8 . The shear crack in the weak layer develops from 6.67 seconds at the time of the maximum amplitude to 6.73 seconds when the shaking direction turns over, and it is found to be the state that the slip surface formed.

As for the horizontal acceleration shown in figure $7 b$ ), the fifth wave amplitude becomes smaller than the amplitude of the input acceleration. It is thought that the action acceleration transmitted the sliding lump has been decreased by the formation of the slip surface, and that the response acceleration in the surface layer became small than the amplitude of the input acceleration. The movement increases after the fifth wave shaking where the slip surface was formed as shown in figure 7a), b), and the acceleration amplitude decreases for the amplitude same as the fifth wave, and It is thought that the slide of the sliding lump occurs.

Then, focusing on negative acceleration about case 9, the movement around $5 \mathrm{~mm}$ occurred to the surface layer near 23.51 seconds when the maximum amplitude occurred as shown in figure 9a), and the tension crack at the shoulder of the slope and the shear crack in the weak layer generate discretely as shown in figure $8 b$ ). The shear crack in the weak layer progresses on 24.27 seconds of $500 \mathrm{Gal}$. Then, it is thought that the slip surface was almost formed afterwards. The horizontal acceleration decreases slightly near 23.51 seconds when the input acceleration becomes minimum as show in figure 9b). However, the negative acceleration amplitude may be small, the movement does not occur at 24.27 seconds after the slip surface was formed. Focusing on the acceleration amplitude when slip surface forms, 900Gal of case 9 exceeds remarkably $600 \mathrm{Gal}$ of case 8 . Focusing on the acceleration amplitude when slip surface forms, 900Gal of case 9 exceeds remarkably $600 \mathrm{Gal}$ of case 8 . Occurrence of such crack can't observe until the maximum amplitude of $800 \mathrm{Gal}$ beyond $600 \mathrm{Gal}$. It points out that the duration as well as the acceleration amplitude is also important about the occurrence of the tension crack and the shear crack.

In collapse mode of slow sliding, the slip surface is formed by the occurrence and the progress of a discrete shear crack from the occurrence of the tension crack as above in the shaking of the acceleration half wave to the negative direction. Moreover, in a formation process of the slip surface, the movement occurs to the sliding lump, and the behavior that the amplitude of response acceleration in the surface layer decrease continues it every negative shaking. Furthermore, the sliding lump 


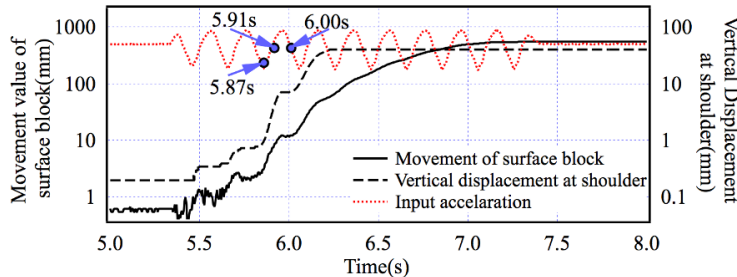

a) Time histories of sliding lump movement

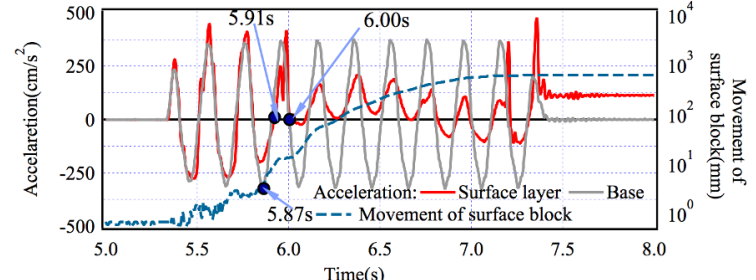

b) Time histories of acceleration at sliding lump and base

Figure 11 Case 5 : sliding lump movement and acceleration at sliding lump and base

( Inclination of weak layer 40 degree, Sinusoidal wave )
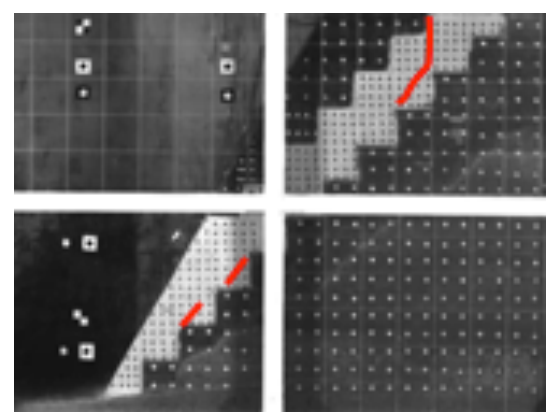

a) $5.87 \mathrm{~s}$
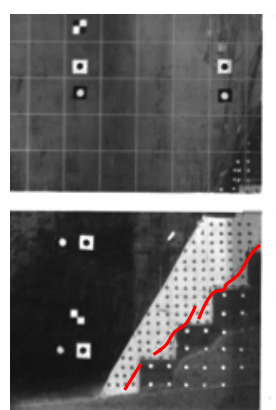

b) $5.91 \mathrm{~s}$
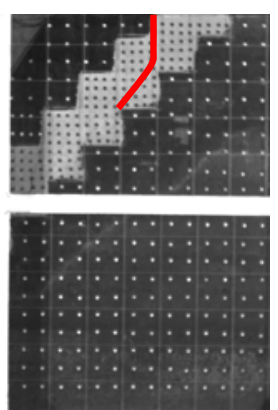

s
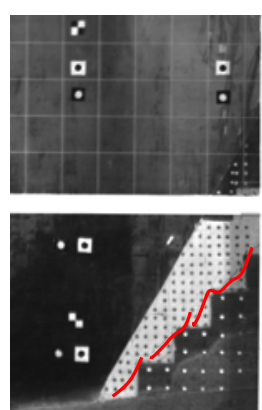

c) $6.00 \mathrm{~s}$
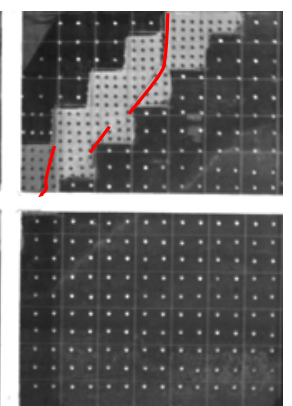

Figure 12 Crack development process of the weak layer for case 5

stands still after the shaking, and it is the characteristic of slow sliding type to be stabilized again.

\subsection{Quick sliding}

As for the results of the last shaking step about case 5 (sinusoidal wave) that the inclination of the weak layer is 45 degrees, time histories of the mean value of the movements obtained by the image analyses about 3 target points in surface layer are shown in figure 11. Time histories of input acceleration at the bottom of the slope model are shown in the same figures. Next, the comparison of the horizontal acceleration time history in the surface layer and that in the base layer is shown in figure 11. Time histories of movement shown in previous figures are shown in the same figures. Finally, the crack development process in the weak layer according to the developmental process of the movement of the sliding lump is shown in figure 12.

The negative first wave of the input acceleration caused the movement as shown in figure 11a), and the movement after the negative third wave increased. The slip surface was formed by the negative process that went to the fourth wave. Just after that, the horizontal acceleration in the surface layer became to 0 as shown in figure $11 \mathrm{~b})$. It is thought that the state that the horizontal acceleration in the surface layer becomes 0 reached the sliding down state that the input acceleration is not transmitted to. For 5.87 seconds at the time of the negative maximum amplitude of the third wave, a shear crack around the toe of weak layer and the tension crack occur as shown in figure 12a). The width of the tension crack grew large as shown in figure $12 \mathrm{~b}$ ) for 5.91 seconds when the shaking direction of the third wave changed, and the shear crack in weak layer progressed to the central part of the weak layer. The shear crack in the weak layer progresses as shown in figure 12c) in 6.00 seconds toward the fourth wave negative shaking, and it is found that the slip surface formed.

Due to not half wave by the horizontal acceleration that the inertial force acts to the toe direction of the slope, but plural half waves, the slip surface is formed by the occurrence and the progress of a discrete shear crack from the occurrence of the tension crack as above. The movement in the surface layer increases in the slip surface formation process, and slide resistance of the ground on the slip surface suddenly decreases, and it may be said that it is a characteristic of collapse mode 'quick sliding' that sliding down occurs.

\section{Applicability of the Newmark method to the behavior of the sliding lump}

Newmark method is a method to calculate sliding displacement of the sliding lump during earthquake. It is based on two assumptions of a sliding lump being rigid and the relationship between stress and stress in the slip surface being rigid plasticity. As for this method, by using the modified Fellenius method as stability analysis method, the slip surface is obtained when safety factor becomes 1.0. Then, it is the method of the idea that slide displacement occurs by an inertial force of the action acceleration after the time when a safety factor equals to 1.0. Furthermore, the peak strength is used as the strength property in the weak layer when a safety factor is either equal to 1.0 or more than 1.0 , and the residual strength is used when a safety factor becomes less than 1.0. This Newmark method applies to the following five cases among the experiment in this paper: Case 6,8 and 11 for slow sliding as the collapse mode, Case 7 and 10 for quick sliding after slow sliding. Because the yield acceleration (= yield seismic coefficient $*$ gravity acceleration) which 


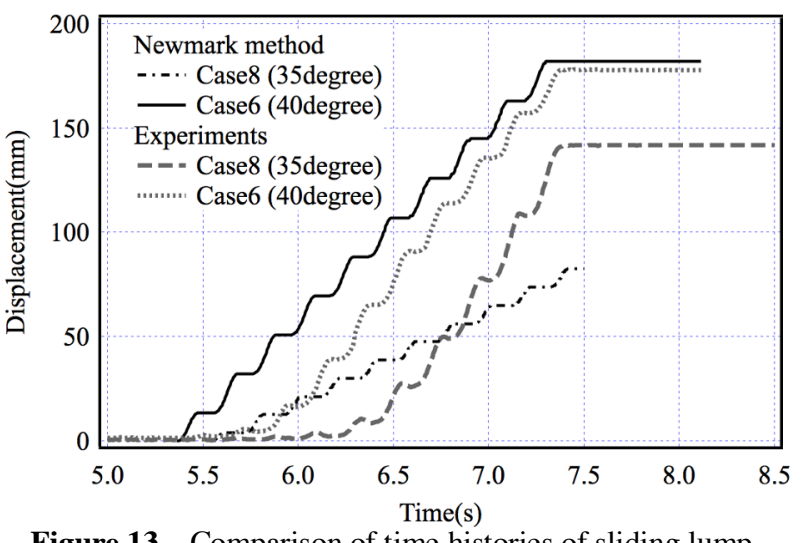

Figure 13 Comparison of time histories of sliding lump by experiment with those by Newmark method

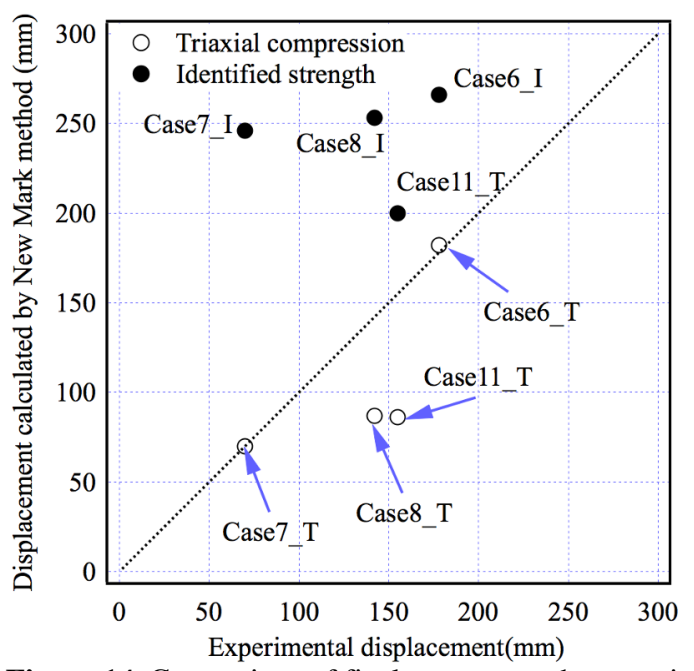

Figure 14 Comparison of final movements by experiment and those by Newmark method obtained by the seismic stability analysis using the peak strength of the weak layer becomes at the same value with the acceleration amplitude for the case by sinusoidal wave shaking, the application of the Newmark method is enabled without calculating the dynamic response of the slope model.

At first, the time histories of the movement of the sliding lump which were obtained from the experiment and Newmark method to the last step of shaking are compared with case 6 about 8 and are shown in figure 13 . Deformation of the weak layer before slip surface formation is included in the movement of two cases here, but it's small compared with the movement. The movement by the experiment is late considerably from the time when the movement calculated by Newmark method occurred. As for Newmark method, the movement of the sliding lump occurs when the safety factor became less than 1.0. However, as for the experiment, the movement of the sliding lump occurs during the process when slip surface is formed after the non-linearization of the weak layer progresses. By this difference, the movement by the experiment is delayed in comparison with movement calculated by Newmark method. Furthermore, focusing on the movement velocity of the sliding lump, the experimental value for case 6 of the inclination of weak layer with 40 degrees is almost the same with that by Newmark method. The movement velocity by Newmark method for the case 8 of the inclination of the weak layer incline with 35 degrees is smaller than that for the case 6 , and the difference in inclination appears definitely. However, as for case 8 , the movement velocity by the experiment is larger than that by Newmark method. The movement about the experiment case of the collapse mode 'slow sliding' that the behavior is almost close to the principle of the Newmark method does not appear the influence of the weak layer inclination and is different from the result by the Newmark method.

Next the comparison of the experimental value and the calculated value by Newmark method is shown in figure 14 about the final displacemt of the sliding lump. As for the final displacement by the Newmark method, the values calculated by using the residual strength obtained from the triaxial compression tests are shown by white circles in the figure. Furthermore, the values calculated by using the residual strength (Internal friction angle : 36.3 degree, Cohesion :1.0 kPa) obtained by inverse analysis of case 5 are shown by black circles in the figure and 2 evaluation values were compared. Here, the diplacement just before sliding down is used as the experimental value about case 7. As for the last movements calculated by using the residual strength obtained from the triaxial compression tests, the values about case 6 and 7 for the inclination of the weak layer with 40 degrees are the same with the experimental values. The values about case 8 and 11 for the inclination of the weak layer with 35 degrees become $0.55-0.60$, i.e. the dangerous evaluation, of the experimental values. As for the last movements calculated by using the residual strength obtained from the inverse analysis of case 5, the values except the value for case 14 become $1.30-3.50$, i.e. lager evaluation, of the experimental values.

Newmark method is the method based on the simple assumption. It is found that the collapse process cannot be expressed appropriately as well as the slide behavior, and that the evaluation value is greatly different from experimental value depending on the strength property of ground material even in the experiment that the location of the slip surface and the response acceleration are clear, among the conditions necessary for the application.

\section{Concluding remarks}

As for the main subject in this paper, first the behavior of the slope until the instability from the stability based on the experimental results of ten cases adding the similar experiments to the existing experiments of the slope models by the shaking table tests under $1 \mathrm{G}$ field, is analyzed and the applicability of Newmark method as analysis of the behavior beyond the stability limit is carried out. The experimental result as the slope behavior was put in order based on collapse mode in the slope 
where Shinoda et al defined and the relation between the formative process of slip surface according to the collapse mode and the response characteristics of the sliding lump was verified. There are several existing study cases about the applicability of Newmark method, but there are no cases which were inspected through the process to a sliding lump's moving from the formation of the slip surface, and it can be said useful knowledge. The following results are found.

- The occurrence time of the movement by the experiment is considerably late to that by the Newmark method, and the movement velocity of the sliding lump by the experiment does not indicate the influence of the weak layer incline that Newmark method appears conspicuously. The movement of the sliding lump by the experiment was also different from the result by Newmark method in the experimental case of the collapse mode 'slow sliding' which indicates the behavior near a principle of Newmark method, and it could be confirmed once more that behavior to slide can't be expressed appropriately from slip surface formative process.

- The final movement value calculated by Newmark method is greatly different from the experimental value depending on the strength property of ground material even in the experiment that the location of the slip surface and the response acceleration are clear, among the conditions necessary for the application.

\section{Acknowledgment}

This research gathered result of "the examination about the damage criterion in the stability evaluation of the slope" that Railway Technical Research Institute (RTRI) was commissioned by the former Japan Nuclear Energy Safety Organization (JNES) in 2008 and 2010. We are grateful to the valuable opinions received from the members of JNES and RTRI.

\section{References}

1. Ministry of Land, Infrastructure and Transport Railway Bureau under the supervision, Railway Technical Research Institute under the compilation, Design Standards for Railway Structures and Commentary (Earth Structures), Maruzen Co., Ltd, 2007(in japanese)..

2. Ministry of Land, Infrastructure and Transport Railway Bureau under the supervision, Railway Technical Research Institute under the compilation, Design Standards for Railway Structures and Commentary (Seismic Design), Maruzen Co., Ltd, 2012(in japanese).

3. Japan road association, Road earth works Embankment works guideline, Maruzen Co., Ltd, 2010(in japanese)..

4. Ministry of Land, Infrastructure and Transport River Burea, Draft of Dam earthquake performance verification guideline against a large-scale earthquake, 2005(in japanese).
5. Newmark N.M. : Effects of earthquakes on dams and embankment, Geotechnique, Vol.15, No.2, pp.139-160, 1965.

6. Shinoda, M., Watanabe, K., Sanagawa, T., Abe, K., Nakamura, H., Kawai, T. and Nakamura, S. : Dynamic behavior of slope models with various slope inclinations, Soils and Foundations, Vol.55, No.1, pp. 127-142, 2015.

7. Masaoki Murata, Hidetaka Nakamura, Masahiro Shinoda, Kenji Watanabe, Taisuke Sanakawa, Tadashi Kawai, Susumu Nakamura, Consideration about a development process of the strain in the rock slope during earthquake, Annual conference of geotechnical engineering, pp.1607-1608, 2011 (in japanese).

8. Hidetaka Nakamura, Masaoki Murata, Masahiro Shinoda, Taisuke Sanakawa, Tadashi Kawai, Susumu Nakamura, Consideration about the seismic deformation property of the rock slope model according to the characteristics of the slip occurrence layer Annual conference of Japan society of civil engineers, III-287, pp.573-574, 2011 (in japanese).

9. Kenji Watanabe, Experimental Technique to Evaluate the Dynamic Deformation Characteristic of Ground, Geotechnical Engineering Magazine, 62(9), pp.20-23, 2014 (in japanese). 